\title{
Questes
}

Revue pluridisciplinaire d'études médiévales

\section{Quelques pistes de réflexion pour une étude scatologique}

\section{Francesco Montorsi}

\section{(2) OpenEdition}

Édition électronique

URL : http://journals.openedition.org/questes/2645

DOI : 10.4000/questes.2645

ISSN : 2109-9472

Éditeur

Les Amis de Questes

Édition imprimée

Date de publication : 15 juin 2011

Pagination : 35-54

ISSN : 2102-7188

\section{Référence électronique}

Francesco Montorsi, «Quelques pistes de réflexion pour une étude scatologique », Questes [En ligne], 21 | 2011, mis en ligne le 15 janvier 2014, consulté le 30 avril 2019. URL : http:// journals.openedition.org/questes/2645; DOI : 10.4000/questes.2645 


\section{Quelques pistes de réflexion pour une étude scatologique}

\section{Francesco MONTORSI}

Certains sujets nécessitent plus que d'autres d'être justifiés et l'étude de la matière fécale dans la littérature est sans doute de ceux-ci. Le thème, convenons-en, peut paraître scabreux. À qui voudrait aborder ce sujet on pourrait reprocher ce que Béralde, dans une première version du Malade Imaginaire de Molière, reprochait à Monsieur Fleurant, éminent spécialiste en clystères : «Allez, Monsieur, allez, on voit bien que vous avez coutume de ne parler qu'à des culs » ${ }^{1}$.

Il n'est donc pas inutile, nous semble-t-il, de commencer par quelques explications ${ }^{2}$. Nous pourrions mettre en avant - comme pour nous en excuser - un intérêt ludique, les excréments jouant un rôle majeur dans l'univers de la parodie et de l'humour. Une étude sur la matière fécale dans la littérature présente néanmoins des intérêts intellectuels et méthodologiques qui dépassent le cadre restreint d'un amusement. Qu'on donne juste un exemple étonnant: les excréments sont à l'œuvre dans plusieurs cosmologies. D'après celles-ci, tantôt la terre, tantôt les hommes, auraient été formés à partir des déjections divines ${ }^{3}$.

Aussi, notre intention est-elle d'offrir aux lecteurs un rapide panorama de la littérature médiévale française touchant à cette odorante matière, en attendant pour l'avenir que quelqu'un assume la tâche d'écrire

\footnotetext{
${ }^{1}$ Moliere, Le Malade imaginaire, Georges Couton (éd.), Paris, Gallimard, «Folio Classique », 1999, p. 197, n. 1.

${ }^{2}$ Remarquable par sa netteté et son tranchant est la justification théorique d'une étude de ce type exprimée par M. Fleming: «Why study shit? Well, why not?». Cf. Margaret FLEMING, «Analysis of a Four-Letter Word», Maledicta: The International Journal of Verbal Aggression, 1 (1977), p. 173-184.

${ }^{3}$ Cf. Enzyklopädie des Märchens, Rolf Wihelm BREDNICH (dir.), Berlin/New York, Walter de Gruyter, 1975-[en cours], t. IV, entrée « Exkremente ».
} 
cette étude d'ensemble qui nous fait défaut. Cette intervention ainsi conçue, ses buts seront principalement d'indiquer ce que nous considérons comme une lacune dans les études médiévales et d'illustrer les quelques éléments qui pourraient représenter des pistes pour la recherche ultérieure.

Malgré l'accord souvent unanime sur la profusion des thèmes scatologiques dans la littérature française, au-delà des rares travaux épars sur tel ou tel autre texte, on ne trouve pas d'étude qui ait traité l'ensemble du sujet ${ }^{4}$. L'ouvrage de Mikhail Bakhtine sur Rabelais et la culture populaire a fourni d'importantes analyses sur la scatologie médiévale, vue comme un des aspects qui relieraient les écrits de l'humaniste français à la culture du Moyen $\hat{A}_{g e}{ }^{5}$. Néanmoins, ce texte de référence, où le Moyen Âge est analysé en fonction des textes de Rabelais, ne peut aucunement représenter un point d'arrivée pour les études médiévales. Il est d'ailleurs surprenant de constater que l'ouvrage de l'érudit russe n'a pas ouvert dans ce domaine la voie aux nombreuses recherches que son accueil en France pouvait laisser attendre. Dans le même temps, de l'autre côté de l'Atlantique, mais aussi plus près, au-delà de la Manche, cette dernière période s'est révélée fructueuses pour les études littéraires scatologiques. Dans l'espace des six dernières années, sont parus des ouvrages aux titres éloquents : Fecal Matters in Early Modern Literature, Excrement in the Late Middle Ages, Medieval Obscenities, On Farting, ainsi qu'un article sur les «fabliaux scatologiques » ${ }^{6}$. Quelques années auparavant, dans son

\footnotetext{
${ }^{4}$ À notre connaissance, la seule tentative de «vue d'ensemble » sur le sujet est l'article d'Albert GIER, « Skatologische Komik in der französischen Literatur des Mittelalters », in Werner SCHRÖDER (dir.), Wolfram-Studien, 7, 1982, p. 154-183.

${ }^{5}$ Cf. Mikhaïl BAKHTINE, L'Euvre de François Rabelais et la culture populaire au Moyen Âge et sous la Renaissance, Paris, Gallimard, 1990 (1 ${ }^{\text {ère }}$ éd. Gallimard 1970/1 ${ }^{\text {ère }}$ éd. russe 1965).

${ }^{6}$ Cf. Fecal Matters in Early Modern Literature and Art. Studies in Scatology, Jeff PERSELS et Russell GANIM (dir.), Aldershot, Ashgate, 2004 ; Susan Signe MorRISON, Excrement in the Late Middle Ages : Sacred filth and Chaucer's fecopoetics, New York, Palgrave Macmillan, 2008 ; Nicola F. MCDONALD, Medieval Obscenities, York, York Medieval Press/Boydell Press, 2006; Valerie ALLEN, On Farting : Language and
} 
ouvrage sur les fabliaux, Howard Bloch avait déjà traité à plusieurs reprises le sujet ${ }^{7}$ et il était allé jusqu'à avouer que l'inspiration pour sa célèbre étude sur les fabliaux lui venait de la lecture de l'un des récits scatologiques de son corpus ${ }^{8}$.

Face à ce foisonnement d'études anglo-saxonnes, force est de constater le peu d'intérêt que les études scatologiques suscitent en France. Il se peut qu'ici une plus grande pruderie décourage certaines approches. Si l'interdit sur la sexualité a commencé à s'écrouler après 1968, ouvrant la voie aux études sur l'expression sexuelle au Moyen Âge, cela n'est pas advenu pour la scatologie, tabou visiblement plus résistant que les autres ${ }^{9}$. Pour s'en rendre compte il est instructif d'ouvrir le Grand Larousse Universel $^{10}$. À l'entrée «scatologie», on lit cette bien mince définition : « Propos ou écrits grossiers où il est question d'excréments ». C'est là sans doute une définition qui, en se focalisant sur la qualité «grossière» de la lettre, évince une partie des sens possibles du récit scatologique ${ }^{11}$. Pour leur part, les études médiévales ne bénéficient guère d'une approche plus

Laughter in the Middle Ages, Basingstoke, Palgrave Macmillan, 2007; Sheila J. NAYAR, "Coprus [sic] Christi: the Scatological Tales of the Fabliaux », in Holly A. CROCKER (dir.), Comic Provocations : Exposing the Corpus of Old French Fabliaux, New York, Palgrave Macmillan, 2006, p. 63-81.

${ }^{7}$ Cf. R. Howard BLoch, The Scandal of the Fabliaux, Chicago/Londres, University of Chicago Press, 1986, notamment les pages 50-54 et 84-86.

8 "It was, I admit, a reading of "De la Crote" against wide-spread claims of the fabliaux's so-called "historical" or "social realism" that first set in motion the train of thought leading to the present book. » ("C'était, je l'avoue, une lecture de La Crote s'opposant à la thèse généralisée d'un prétendu "réalisme historique" ou "social" des fabliaux qui a déclenché la chaîne de raisonnements qui a amené à ce livre »), ibid., p. 58 .

9 L'une des raisons de la résistance de ce tabou «scientifique» semble d'ordre psychologique : aucun chercheur ne veut que son nom soit lié à cette matière.

${ }^{10}$ Grand Larousse Universel, Paris, Larousse, 1991, 15 vol., entrée « scatologie ».

11 Or, la scatologie est à l'œuvre dans des mythes fondateurs de plusieurs peuples.

Cf. Enzyklopädie des Märchens, op. cit. 
étendue de la question. Dans les dictionnaires et les encyclopédies portant sur le Moyen Âge, on ne trouve pas d'entrée consacrée à la scatologie ${ }^{12}$.

\section{Les excréments dans l'histoire}

Avant d'établir notre corpus et, ensuite, d'analyser nos textes, il nous faut mettre en évidence un axiome tout à fait banal : le rapport de l'homme à ses excréments évolue dans le temps. Ce qui, il n'y a pas plus de quatre cents ans, était normal, est considéré aujourd'hui comme rebutant. Le premier savant qui a examiné les pratiques liées à l'excrétion dans le contexte des changements sociaux et culturels sur la longue durée est Norbert Elias. Dans son ouvrage, La Civilisation des mours ${ }^{13}$, le sociologue allemand a été amené à constater qu'au Moyen Âge le commerce de l'homme avec ses fèces était sans doute moins normé qu'aujourd'hui :

12 Cf. Dictionary of the Middle Ages, Joseph R. STRAYER (éd.), New York, Charles Scribener's sons, 1982-1989, 13 vol.; Lexicon des Mittelalters, Munich/Zurich, Artemis/Lexma, 1977-1999, 10 vol.; Dictionnaire encyclopédique du Moyen Âge, André VAuchez (éd.), Paris, Le Cerf, 1997, 2 vol. Bien qu'elles ne soient pas consacrées au Moyen Âge, nous pouvons lire deux entrées intéressantes dans l'Enzyklopädie des Märchens, op. cit., t. IV, entrée «Exkremente» et t. XII, entrée «Skatologie ». Albert Gier, auteur de l'entrée «Skatologie», se plaint de la situation lacunaire des instruments de recherche (p. 762) : «Die intensive Bachtin-Rezeption in der Literaturwissenschaft hat zwar zahlreiche Einzelstudien zu Skatologie und Obszönität speziell in Schwankerzählungen, aber keine systematische Untersuchungen hervorgebracht; bis heute fehlt ein Artikel "Skatologie" in fast allen literaturwissenschaftlichen Enzyklopädien » («L'intense réception de Bakhtine dans la littérature scientifique a produit de nombreuses études isolées sur la scatologie et l'obscénité, spécialement dans les fabliaux, mais aucune recherche systématique. À l'heure d'aujourd'hui une entrée "Scatologie" fait défaut dans presque toutes les Encyclopédies littéraires »).

${ }^{13}$ Norbert EliAs, La Civilisation des mours, Paris, Calmann-Lévy, 1973. David Inglis a poursuivi cette analyse sociologique de la défécation : cf. David INGLIS, A Sociological History of Excretory Experience. Defecatory Manners and Toiletry Technologies, Edwin Mellen, Lampeter, "Mellen Studies in Sociology », 30, 2001. Les thèses d'Elias ont été contestées par Hans Peter DUERR dans Nudité et Pudeur : le mythe du processus de civilisation, Paris, Édition de la Maison des sciences de l'homme, 1998 (1 ${ }^{\text {ère }}$ éd. allemande 1988). 
$\mathrm{Au}$ début, les fonctions du corps et leur vue ne s'accompagnaient que peu de sentiments de pudeur et de gêne ; par conséquent on ne songeait guère à les entourer de discrétion ou à les réprimer. ${ }^{14}$

Au Moyen Âge, les excréments n'étaient pas confinés dans l'espace clos et séparé qu'on leur réserve aujourd'hui. Bien au contraire, ils envahissaient l'espace public. Les toilettes privées, très rares, étaient des fosses à ciel ouvert et les fosses d'aisances disséminées dans la ville étaient souvent des solutions de fortune. Les lieux où se libérer pouvaient d'ailleurs être des plus improvisés, et on sait même que certains culs-de-sac, souvent à proximité du marché, étaient tout naturellement destinés à cette besogne ${ }^{15}$. Les rues retentissaient des fameux cris «Gare l'eau!» qui devaient prévenir les malchanceux passants du jet des eaux usées, des ordures les plus diverses, parfois même des matières fécales. Au XVI ${ }^{\mathrm{e}}$ siècle à Paris se succédaient encore des ordonnances - avec quel résultat? - pour interdire aux Parisiens de jeter leurs ordures sur la place publique. Parmi des bois gravés publiés en 1582 représentant les crieurs parisiens, nous trouvons une mystérieuse figure. Armé de seaux dissimulés sous un vaste manteau, le personnage fait retentir un cri énigmatique dans la ville : « Ie sçay bien ce que sçay faire ». Métier vraiment ingrat: cet homme est un chalet de nécessité ambulant ${ }^{16}$.

Les hommes du passé ne voyaient pas dans leurs déjections les répugnants résidus des processus de l'organisme que l'on y voit

\footnotetext{
${ }^{14}$ Norbert EliAS, op. cit., p. 195.

${ }^{15} \mathrm{Cf}$. Georges Vigarello, Le Propre et le sale. L'Hygiène du corps depuis le Moyen Âge, Paris, Seuil, « Points », 1985, p. 66 et Roger-Henri GuERRAND, Les Lieux. Histoire des commodités, Paris, La Découverte, 2005 (1 ${ }^{\text {ère }}$ éd. 1985), p. 16-17.

${ }^{16}$ Cf. Robert MAssin, Les Cris de la ville, Paris, Gallimard, 1978, p. 30. Le quatrain qui accompagne le bois gravé est fort instructif : «Avec un long manteau i'alloy par ceste ville / Et portoy deux grands seaux où l'on chioit debout : / Mais voyant auiourd'huy que l'on chie partout, / Ie ne m'en mesle plus : l'office est inutile ».
} 
aujourd'hui ${ }^{17}$. Les exemples de l'estime dans laquelle les fèces pouvaient être tenues dans les époques antérieures pourraient être multipliés à foison. D'après le témoignage de l'Histoire naturelle, les patriciennes romaines se seraient maquillées, entre autres, avec des crottes de rat ${ }^{18}$. En outre, à l'époque de Pline, les déjections d'animaux étaient employées aussi dans la préparation des médicaments et cela a continué au moins jusqu'au XIX ${ }^{\mathrm{e}}$ siècle dans la médecine populaire ${ }^{19}$ : encore à la fin du $\mathrm{XVII}^{\mathrm{e}}$ siècle, un médecin allemand pouvait écrire un livre très sérieux sur les médicaments qui peuvent être produits à partir de cette vile matière ${ }^{20}$. Enfin, d'après les analyses des ethnographes, plusieurs tribus ont un rapport rituel avec les excréments ${ }^{21}$ et cet aspect se retrouve dans certaines fêtes populaires qui ont été célébrées jusqu'à la Révolution industrielle ${ }^{22}$. Dans ces cérémonies, nous aurions pu voir des phénomènes tels que la projection d'excréments et l'arrosage d'urine, ce dernier étant aussi un thème récurrent dans l'œuvre

\footnotetext{
${ }^{17}$ Un lien entre la perception moderne des excréments et la représentation capitaliste du cycle productif est établi par Nicole BELMONT, entrée «Escrementi », in Ruggiero ROMANO (dir.), Enciclopedia Einaudi, Turin, Einaudi, 1977-1984, t. V, p. 765-776 (p. 776): «La polluzione è l'escrementizio tale quale lo vede fallacemente il capitalismo : puro scarto non riciclabile, non biodegradabile, mentre gli escrementi costituiscono una fase del ciclo vitale » («La pollution relève de l'excrémentiel tel que le voit erronément le capitalisme : simple déjection non recyclable, non biodégradable, tandis que les excréments constituent une phase du cycle vital »).

${ }^{18}$ Pline L'ANCIEN, Histoire Naturelle, Paris, Les Belles Lettres, 1950-1972, 37 vol., livre XXX, x, p. 33.

19 Cf. Nicole Belmont, entrée «Escrementi», in Ruggiero Romano (dir.), Enciclopedia Einaudi, op. cit, p. 772-773. Au XVI ${ }^{\mathrm{e}}$ siècle Martin Luther, comme beaucoup d'autres, était persuadé de la valeur thérapeutique des excréments. Cf. Les Propos de table, Gustave BRUNET (éd. et trad.), Paris, Frères Garnier, 1844, p. 377 : « Je suis surpris que Dieu ait mis dans la fiente des remèdes si importants et si utiles, car l'on sait par expérience que la fiente de truie arrête le sang, et celle de cheval sert pour la pleurésie. La fiente de l'homme guérit les blessures et les pustules noires [...]. »

${ }^{20}$ Cf. Christian Franz PAULINI, Heylsame Dreck-Apothecke : wie nemlich mit Koth und Urin die meisten Krankheiten und Schäden glücklich geheilet worden, Stuttgart, Verlag des Herausgebers, 1847 ( $1{ }^{\text {ère }}$ éd. allemande 1696).

${ }^{21}$ Cf. John Gregory Bourke, Les Rites scatologiques, Dominique G. LAPOrTE (éd.), Paris, PUF, 1981 (1 1 ère éd. américaine 1891).

${ }^{22}$ Bakhtine parle aussi de ces fêtes. Cf. Mikhaïl BAKHTINE, op. cit, p. 13, 83-91, 150 et passim.
} 
littéraire de Rabelais ${ }^{23}$. Sans cette connaissance préalable, ce passage du Roman de Fauvel, l'une des premières descriptions du charivari, nous semblerait énigmatique :

L'un cassoit fenestres et huis,

L'autre getoit le sel ou puis,

L'un getoit le bren aus visages. ${ }^{24}$

\section{Repères méthodologiques}

Avant de jeter les bases d'une investigation scatologique, il faut établir sa méthode et ses instruments. Il est d'abord nécessaire de définir ce qu'on entend par «scatologie». La scatologie concerne en théorie les excréments de l'homme. Néanmoins, elle peut s'occuper de tous les écarts $\mathrm{du}$ corps humains (urine, pets, etc.) ${ }^{25}$. Les occurrences de «pets» et d' « urine » dans la littérature médiévale étant en nombre très élevé, nous nous sommes ici limité à l'étude des excréments.

Deuxième étape : comment établir le corpus? D'abord, nous pouvons nous servir de dictionnaires et de glossaires de l'ancienne langue. Pour ce faire, il est fondamental de déterminer le champ sémantique de la scatologie au Moyen Âge. Certains mots n'ont pas changé («merde», «chier », « crotte », « étron », « fumier », etc.). D'autres ont disparu : c'est le cas de «eschiter», «bren» (qui, n'est plus d'usage sauf dans certains

\footnotetext{
${ }^{23}$ L'épisode le plus connu est celui où Pantagruel inonde les habitants de Paris : « Lors [Gargantua] en soubriant destacha sa belle braguette, et tirant sa mentule en l'air les compissa si aigrement, qu'il en noya deux cens soixante mille, quatre cens dix et huyt. Sans les femmes et petiz enfans. », François RABElaIS, Gargantua, in CEuvres complètes, Mireille HuCHON (éd.), Paris, Gallimard, «Bibliothèque de la Pléiade », 1994, chap. XVII, p. 48). Un autre exemple se trouve au chap. XXXVIII, p. 105 : « Lors [Gargantua] pissa si copieusement, que l'urine trancha le chemin aux pelerins et furent contrainctz passer la grande boyre $\gg$.

${ }^{24}$ Gervais du Bus, Roman de Fauvel, Arthur LÅnfors (éd.), Paris, Firmin Didot, 1914-1919, p. 166, v. 737-739. L'éditeur interprète «bren» comme «partie la plus grossière du son ». Il semble préférable ici de traduire le mot par "merde », comme le fait Claude LECOUTEUX dans la traduction qu'il fournit dans Chasses fantastiques et cohortes de la nuit au Moyen Âge, Paris, Imago, 1999, p. 148-149.

${ }^{25}$ Cf. Enzyklopädie des Märchens, op. cit., t. XII, entrée «Skatologie », p. 761.
} 
emplois régionaux, par exemple en picard), «fien », « esmelcier », « laisse », « esmeut », « foire » (dont on a conservé les mots dérivés). Nous remarquons tout de suite que le changement peut-être le plus marquant a été opéré dans la sélection du lexique, aujourd'hui moins riche et varié qu'il ne l'était au Moyen Âge ${ }^{26}$. Rabelais, au $\mathrm{XVI}^{\mathrm{e}}$ siècle encore, montre une profusion de mots orduriers qui aujourd'hui nous fait défaut :

Ha, ha, ha ? Houay ? Que Diable est cecy ? Appellez vous cecy foyre, bren, crottes, merde, fiant, dejection, matiere fecale, excrement, repaire, laisse, esmeut, fumée, estront, scybale ou spyrathe ${ }^{27}$

Une fois cernés nos objets linguistiques, il faut opérer une division et traiter la matière brute. Nous avons beaucoup de «gros mots », parmi lequels «merde » et compagnie ont la part belle ${ }^{28}$. Néanmoins, il ne faut pas s'arrêter longtemps sur le succès de ces deux syllabes qui, en France, ont eu la gloire d'ouvrir des pièces et d'immortaliser des moments historiques. Dans ce type d'occurrences, le mot, employé comme juron, est vidé de son véritable référent et il paraît légitime de ne pas s'y attarder. En effet, pour qu'une occurrence soit prise en considération, elle doit assumer une fonction narrative. En d'autres termes, pour qu'il y ait scatologie, il ne suffit pas d'une référence quelconque aux excréments; il faut aussi que ces excréments jouent un rôle narratif dans le texte, qu'il y ait ce qu'on pourrait appeler une « fonction scatologique ».

\footnotetext{
${ }^{26}$ Gaston Phébus dans le Livre de la Chasse nomme les excrétions de plusieurs types d'animaux. Nous avons «fumee », « laisse », « crotes », « fiante » et « espreintes ». Cf. Gaston Phebus, Livre de la Chasse, Gunnar Tilander (éd.), Stockholm/Paris, Almquist et Wiksells/Crépin-Leblond, 1971, chap. XXX, p. 156.

${ }^{27}$ François RABELAIS, Le Quart Livre, in Euvres complètes, éd. cit., chap. LXVII, p. 701.

${ }^{28}$ Cf. Pierre GuIRAUD, Les Gros mots, Paris, PUF, « Que sais-je? », n¹579, 1975, en particulier les pages 94-99 consacrées au mot «merde », "l'un des mots clés de la langue française ». Pour les insultes au Moyen Âge, voir Nicole GONTHIER, "Sanglant Coupaul!» "Orde Ribaude!» Les injures au Moyen Âge, Rennes, Presses Universitaires de Rennes, 2007.
} 
Nous avons décidé de travailler sur un corpus essentiellement comique, constitué par plusieurs fabliaux, un conte à rire en vers qui a été parfois apparenté aux fabliaux, et deux épisodes de la saga de Renart. Nous ne nous attardons donc pas sur les occurrences des excréments dans la chanson de geste (qui serait pourtant un objet d'étude très intéressant) ${ }^{29}$ ou dans les romans ${ }^{30}$. Il est évident que la fonction scatologique se trouve de manière prépondérante dans des textes à contenu comique. En effet, la transgression de tabous puissants, comme celui qui pèse sur l'excrémentiel, s'accompagne souvent, dans la vie quotidienne aussi bien que dans la littérature, d'un rire libérateur dont la fonction est de remplacer la honte. Le corpus que nous nous proposons d'étudier est, pas conséquent, représentatif de textes vulgaires « à rire » des $\mathrm{XII}^{\mathrm{e}}$ et XIII ${ }^{\mathrm{e}}$ siècles. Les fabliaux que nous retiendrons sont Charlot le Juif, Trubert, Jouglet, La Crote, Le Vilain Asnier et Porcelet. Quant au Roman de Renart et ses annexes, nous lirons, parmi d'autres passages possibles, le Couronnement de Renart et la XVII branche (selon la numérotation de l'édition d'Armand Strubel). Enfin, nous trouverons un texte comique, souvent considéré comme une parodie de chanson de geste, Audigier.

\section{Une brève lecture du corpus}

Passons maintenant à un rapide survol des textes de notre corpus. Compte-tenu des limites imposées à cette communication, nous nous

\footnotetext{
${ }^{29}$ Qu'on pense au comte Tiébaut qui se souille de peur dans la Chanson de Guillaume. Cf. La Chanson de Guillaume, François SuARD (éd.), Paris, Classiques Garnier, 1999, p. 24, v. 345-348.

${ }^{30}$ La littérature arthurienne n'est pas exempte de références aux excréments. Dans le Perceforest, le chevalier Estonné trébuche dans un tas de fumier. Cf. Perceforest, Gilles Roussineau (éd.), Paris, Droz, 1999, $2^{\mathrm{e}}$ partie, t. I, p. 165.
} 
limiterons à de brèves observations sur le rôle de la fonction scatologique dans chaque texte ${ }^{31}$.

S'il y a un texte scatologique par excellence au Moyen Âge, c'est bien Audigier ${ }^{32}$, un conte à rire dont toute la trame est construite sur une exhibition complaisante et grotesque des excréments. Dans la mesure où Chloé Chalumeau propose une étude de ce texte dans ce bulletin, nous ne nous attarderons pas sur cette œuvre ${ }^{33}$. Nous retiendrons simplement que, de tous les textes du corpus scatologique, celui-ci est le seul qui reste énigmatique à tous points de vue. Limitons-nous à citer l'incipit, dont la tonalité permettra au lecteur de se faire aisément une idée du reste du récit :

Tel conte d'Audigier qui en set pou,

Mais ge vos en dirai trusqu'a harou.

Ses peres tint Cocuce, i. païs mou,

Ou les genz sont en merde jusques au cou :

Par un ruissel de foire m'en ving a nou,

Onques n'en poi oissir par autre trou. ${ }^{34}$

Intéressons-nous plutôt aux aventures de l'animal le plus habile et le moins honnête de toute la faune médiévale. Dans les deux ouvrages, nous assistons à une scène assez semblable. Dans la branche XVII, Renart cherche, comme souvent, un moyen d'apaiser sa faim. La chance l'amène devant un berger qui s'est endormi en faisant paître ses bêtes. La ruse de l'infâme animal est d'une grossièreté saugrenue mais, au fond, efficace. Renart grimpe sur l'arbre sous le feuillage duquel le berger se repose :

Renart fist conme pute beste :

Quant il li fu desus la teste,

\footnotetext{
31 Pour la même raison, nous renonçons aussi à fournir la bibliographie relative à chaque œuvre.

32 Denis Joseph ConLON en a fourni une édition dans son article «La Chanson d'Audigier - A Scatological Parody of the Chansons de geste edited from Ms. Bibliothèque nationale, f. fr. 19152 », Nottingham Medieval Studies, XXXIII (1989), p. 21-55.

${ }^{3}$ Voir dans ce bulletin la contribution de Chloé Chalumeau, «La Scatologie dans Audigier : de la chanson de geste au fabliau », p. 55-71.

${ }^{34}$ Audigier, éd. cit., p. 30, v. 1-6.
} 
Dresce la queue et aler laisse

Tout contreval une grant laisse

De faire claire a cul overt.

Tout li en a le vis couvert!

Cius qui l'a sentue s'esvelle:

Tausta son vis et s'esmervelle

Que çou est qui li chiet si chaut $[\ldots]^{35}$

Nous retrouvons dans Le Couronnement de Renart le même motif : « un balourd est conchié par l'animal». Ici, le goupil réussit à tromper un paysan qui vient de capturer Ysengrin grâce à un piège à loups. L'ignoble rouquin s'arrange pour avoir le loup en échange de cent livres mais, ensuite, il entraîne le vilain dans un bourbier où le simplet reste inévitablement coincé. À ce moment, la revanche de Renart est inexorable :

«Vilains, dist il, de ton lovier

Veil or que t'aies le merite. »

Le visage tout li eschite,

Nes et baulevres et menton.

Renars a dit : « Ensi doit on

Le covoiteus tous jors paiier. »

De maintenant li vait raiier

Del compenage c'ot el ventre $[\ldots]^{36}$

Dans ces deux passages, les déjections sont l'instrument d'une humiliation : ils déterminent un violent rabaissement de la victime. De plus, un lien est établi entre les excréments et l'avarice, ce qui n'est pas sans rappeler certaines théories de Freud ${ }^{37}$. Enfin, entre le paysan et les excréments s'instaure un rapport très étroit, un rapport métonymique, en raison de la contiguïté entre vilain et matière fécale, aussi bien que, très

${ }^{35}$ Le Roman de Renart, Armand Strubel (éd.), Paris, Gallimard, « Bibliothèque de la Pléiade », 1998, Branche XVII, Le Partage des proies, v. 973-982.

${ }^{36}$ Le Couronnement de Renard, Alfred Foulet (éd.), Paris/Princeton, PUF/Princeton University Press, 1929, p. 24-25, v. 786-793.

37 Cf. Sigmund FrEUD, «Caractère et érotisme anal» et «Des transpositions pulsionnelles, en particulier dans l'érotisme anal», in CEuvres Complètes, André Bourguignon, Pierre Cotet et Jean LaPlanche (éd.), Paris, PUF, 2007 ( 1 ère éd. allemande 1908), t. VIII, p. 187-194 et t. XV, p. 53-62. Dans la perspective qui est celle de la psychanalyse, l'argent et les excréments s'équivalent et l'avarice apparaît comme une pulsion sublimée de la sexualité anale. 
explicitement, métaphorique. Dans la branche XVII, tout de suite après que Renart a conchié le paysan, Noble le lion prononce une phrase éloquente : « Je n'ai mie vilain tant chier. Autant ameroie a touchier A un ort vessel de ma main Conme je feroie a vilain. ${ }^{38}$

Passons aux fabliaux. La matière fécale est fondamentale dans Charlot le Juif de Rutebeuf. Charlot est un jongleur, sans doute un juif converti, qui participe à un mariage. À la fin de la cérémonie, il ne trouve pas une reconnaissance adéquate de la part du panetier, un petit seigneur, qui devrait le rémunérer : en échange de ses services, l'homme, au lieu de lui remettre de l'argent, lui donne une peau de lièvre sans valeur. La réaction du ménestrel est une ruse d'une puissante simplicité ordurière :

Porpenceiz s'est que il fera

Et comment il li rendera.

Por li rendre la felonie

Fist en la pel la vilonie -

Vos saveiz bien ce que vuet dire.

Arier vint et li dist : «Biau sire,

$\mathrm{Se}$ ci a riens, si le preneiz.

- Or as-tu dit que bien seneiz ?

- Oill, foi que doi Notre Dame!

- Je cuit c'est la coiffe ma fame,

Ou sa toaille ou son chapel :

Je ne t'ai donei que la pel. »

Lors a boutei sa main dedens :

Eiz vos l'escuier qui ot gans

Qui furent punais et puerri

Et de l'ouvrage maitre Horri ! ${ }^{39}$

Premièrement, les excréments sont évoqués par deux euphémismes, d'abord « la vilonie », terme renforcé par une adresse complice au lecteur

${ }^{38}$ Le Roman de Renart, Ernest MARTIN (éd.), Berlin/New York, De Gruyter, 1973 (1 $1^{\text {ère }}$ éd. 1885), t. II, p. 187, v. 1181-1186. L'édition d'Armand Strubel rejette « ort vessel » parmi les variantes et choisit à sa place la leçon « ort mesel ».

${ }^{39}$ RutEBEUF, Charlot le juif et la peau de lièvre, in CEuvres complètes, Edmond FARAL et Julia BASTIN (éd.), Paris, Picard, 1985 (1 ${ }^{\text {ère }}$ éd. 1959-1960), 2 tomes, t. II, p. 256-259. Le passage cité correspond aux vers 111-126. 
(« vous savez bien ce que veut dire »), ensuite «l'ouvrage maitre Horri », expression formulaire d'après le nom propre d'un célèbre vidangeur de Paris $^{40}$. Cette pudeur est particulière à certains auteurs. Il se peut qu'elle soit plus développée chez les écrivains, comme Rutebeuf, qui n'abandonnent pas leur œuvre à la condition d'anonymat. Deuxièmement, les excréments sont encore l'instrument unique d'une ruse, d'un tour joué par un ménestrel à un petit seigneur qui n'a pas accompli le devoir social de la largesse. Les excréments étant - comme nous l'avons déjà souligné une métaphore fréquente de l'argent ${ }^{41}$, l'avarice peut être vue comme une sorte de constipation, d'où le contrappasso scatologique par lequel l'avare, tout comme le «convoiteux» du Couronnement, est puni de son péché. Enfin, le poids de la dépréciation paraît peser en même temps sur le panetier et sur le jongleur. Dans le personnage de Charlot cohabitent en effet deux statuts dévalorisants pour le Moyen Âge, la religion juive et la profession de jongleur. D'ailleurs, Rutebeuf même ne cache pas son manque de considération pour ce personnage « qui n'estoit pas moult biauz valloz $»^{42}$.

Le même lien entre humiliation et matière fécale se retrouve dans Trubert $^{43}$. Le héros de ce fabliau est un digne compagnon de Renart et, n'était sa balourdise, on serait tenté de le comparer au marquis de Sade en raison de sa cruauté. L'intrigue du texte est d'une extrême banalité : grâce à ses déguisements et à ses tours, un paysan dupe à plusieurs reprises un duc. Dans l'une de ses folles initiatives, Trubert se déguise en médecin et arrive

\footnotetext{
${ }^{40}$ Ibid., t. I, p. 557, n. 141.

${ }^{41}$ Qu'on pense au thème folklorique de l'«or déféqué ». Pour ce dernier, voir l'Enzyklopädie des Märchens, op. cit., t. XI, entrée « Puppe : Die beißende P. ».

${ }^{42}$ Rutebeuf, Charlot le juif, éd. cit., p. 258, v. 70.

43 Douin De Lavesne, Trubert, fabliau $d u$ XIII ${ }^{e}$ siècle, Gaston RaYnaud De Lage (éd.), Genève, Droz, 1974.
} 
à conchier le noble avec « un estront de chien ${ }^{44}$ qu'il fait passer pour une médecine :

De son sachet la boiste tret ;

De ce qu'il a dedenz trouvé

Il a le cors oint et doré.

«Deus! dit li dus, biaus rois puissanz,

Com par put or cist oignemenz !

Aussi put com merde de chien!

- Sire, vos devinez mout bien,

Dit Trubert, par tans garirez. ${ }^{45}$

Ce texte inverse donc le rapport hiérarchique propre à la société médiévale, ce qui nous rappelle l'analyse bakhtinienne du « bas corporel » comme principe de renversement des valeurs. En outre, bien que le lien entre excréments et médecine puisse paraître accidentel, il y a des chances que ce thème relève d'une ancienne tradition. Nous avons déjà vu que, des siècles durant, la médecine populaire a exploité les déjections animales pour préparer des médicaments. Le motif des excréments bénéfiques apparaît au Moyen Âge, non seulement dans les traités médicaux, mais aussi dans deux textes littéraires que nous allons citer à l'appui de cette hypothèse ${ }^{46}$. Dans le Dit de l'herberie, Rutebeuf explique à son lecteur comment préparer un onguent, un remède imparable pour une douleur de dents :

Oeiz coument jou confirai ;

Dou confire ne mentirai,

C'est sens riote.

Preneiz dou saÿn de marmote,

De la merde de la linote

Au mardi main,

Et de la fuelle dou plantain,

Et de l'estront de la putain,

\footnotetext{
${ }^{44}$ Ibid., p. 37, v. 1090.

${ }^{45}$ Ibid., p. 43, v. 1258-1265.

${ }^{46}$ Le motif des excréments thérapeutiques se retrouve aussi dans des textes littéraires de la Renaissance. Cf RABELAIS, le Quart Livre, éd. cit., p. 553 et MARGUERITE DE NAVARre, Le Malade, in Théâtre Profane, Verdun-Louis SAULNIER (éd.), Genève, Droz, 1978, p. 1-33 (notamment p. 20).
} 
Qui soit bien ville $[\ldots]^{47}$

Dans le fabliau Le Vilain Asnier ${ }^{48}$, un paysan conduit son chariot chargé de fumier à travers la rue des épiciers de Montpellier. Soudain, suffoquant à cause des effluves s'exhalant des épices, il tombe évanoui. Une petite foule s'inquiète de l'homme allongé sur le terrain quand un passant anonyme, arrivé sur la scène, promet de guérir sur-le-champ le malchanceux ânier moyennant une belle récompense. Inutile de préciser que les excréments, encore une fois, ont la part belle dans ce remède qui se révélera efficace pour le pauvre vilain :

Donc prant la forche qu'il portoit,

A quoi il ses asnes chaçoit ;

Du fien a pris une palee,

Si li a au nes aportee. ${ }^{49}$

Un autre texte doit attirer notre attention : il s'agit du fabliau intitulé La Crote $^{50}$, désigné dans un autre manuscrit sous le nom évocateur de Fabliau de la Merde ${ }^{51}$. Le texte se compose d'une soixantaine de vers et a la structure brève et agile d'une plaisanterie. Le fabliau s'ouvre sur une scène d'intérieur : devant un feu de bois se réchauffe un couple de rustres. L'homme saisit «sa couille et son vit ${ }^{52}$ et invite sa dame à deviner ce qu'il tient dans sa main. La femme ne devine pas, mais riposte par une nouvelle devinette : qu'a-t-elle dans sa main ? demande-t-elle en passant à son mari une petite boule de matière pâteuse. $\mathrm{Si}$, en trois tentatives, le mari trouve la bonne réponse, il aura en échange du vin. Malheureusement, le premier et le deuxième essais de l'homme échouent. La femme s'écrie :

- «Par foi, c'est mençonge provee ! »

\footnotetext{
${ }^{47}$ RuteBeuf, Le Dit de l'herberie, in CEuvres complètes, éd. cit., p. 272-280, v. 77-85.

${ }^{48}$ Le Vilain Asnier, in Nouveau Recueil Complet des Fabliaux, Willem NoomEN et Nico VAN DEN BoOgAard (éd.), Assen, Van Gorcum, 1983-1998, 10 tomes, t. VIII, p. 207-214. Ce recueil est dorénavant cité sous le sigle N.R.C.F.

${ }^{49}$ Ibid., p. 214, v. 37-40.

${ }^{50}$ La Crote, in N.R.C.F., éd. cit., t. VI, p. 25-32.

${ }^{51}$ Paris, BnF, f. fr. 1593, fo $80 \mathrm{r}^{\circ}$.

${ }^{52}$ La Crote, in N.R.C.F., éd. cit., p. 30, v. 13.
} 
Fet cele, qui le tient por sot.

«Or n'avez a dire q'un mot. » ${ }^{53}$

La réaction du mari est résolue. Mais peut-être que, s'il avait su, il n'aurait pas agi ainsi :

Et cil en sa gole dedenz

La masche et mere entre ses denz,

Que paor a que il ne perde.

«Par le sanc Dé, fet il, c'est merde!

Or m'en puis bien apercevoir !

- Par mon chief, vos avez dit voir :

Ce est merde de tot a estrous!

Ja mes ne gaigerai a vos:

Deable vos ont fait devin!

Je vos doi denree de vin. ${ }^{54}$

L'auteur de ce fabliau s'inscrit dans la riche tradition littéraire des paysans vilipendés. La «fonction scatologique» est d'ailleurs employée dans d'autres textes pour s'en prendre aux vilains : qu'on pense au Pet au Vilain de Rutebeuf par exemple ${ }^{55}$.

Nous voilà arrivé à notre dernier texte, le fabliau Jouglet ${ }^{56}$ qui aurait été écrit par Colin Malet, auteur qui nous est connu seulement à travers ce texte. Nous retrouvons ici deux figures déjà rencontrées dans les exemples précédents : un jongleur, Jouglet, et un paysan, Robin. Ce dernier, grâce au truchement de sa mère, convole en noces avec Mahaut, fille d'un vavasseur. Le rustre, ignare des choses du monde, est confié par sa mère au jongleur afin que celui-ci lui enseigne comment se tenir le jour des noces. Pour Jouglet, doué d'un penchant pour la moquerie, un tel lourdaud c'est du pain béni : il en profite pour mettre en pratique tout son talent de gabeur. Il lui est facile de convaincre Robin qu'avant le mariage il est d'usage de

\footnotetext{
${ }^{53}$ Ibid., p. 32, v. 50-52.

${ }^{54}$ Ibid., v. 53-62.

${ }^{55}$ Rutebeuf, Le Pet au Vilain, in CEuvres complètes, éd. cit., t. II, p. 305-308.

${ }^{56}$ Jouglet, in N.R.C.F., éd. cit, t. II, p. 185-214.
} 
manger une énorme quantité de poires, pour ensuite lui interdire de déféquer sous prétexte,

« [...] que l'en ne chie mie

le jor qu'en espouse s'amie,

quer ce seroit trop grande ledure. $»^{57}$

Robin arrive donc à sa première nuit d'amour avec un ventre chargé et un impérieux besoin de se soulager. Mais la jeune mariée comprend le $g a b$ dont son mari et elle sont les victimes et a la vivacité d'esprit, tout aussi bien que le mauvais goût, de retourner la situation au détriment du jongleur. Elle pousse son époux docile à satisfaire sans gêne ses besoins partout où cela peut incommoder le jongleur :

Tant le commencha a proier

Qu'il li dist : « Je muir de chier »,

E qu'eissi l'a Juglet servi.

«Qu'est? Por ceste ne por celui !

Si vos a eissi atorné,

Or tost, n'i ait plus demoré,

Il gist coste cele paroit :

Chiez a son chevez tout droit,

Sor sa chemise en cel espuer $! »{ }^{58}$

Une seule évacuation ne suffira pas à apaiser le drame intestinal de Robin et, à chaque nouvelle crise, la femme ne cessera de l'inciter à conchier tout ce qui appartient au jongleur. Le lendemain, Jouglet sera ainsi couvert de matière fécale, du matin, en se réveillant dans un lit plein d'excréments, jusqu'au soir, quand, en sortant sa vielle de sa boîte, il éclaboussera sans le vouloir deux paysans qui lui paieront le service à grand renfort de coups. Et l'histoire de se terminer sur une moralité :

De cel an ne se pout aidier. Eissi fu conchié Juglet !

Segnors, ce dit Colin Malet,

Tel cuide conchier autrui

Qui assez miez conchie lui. ${ }^{59}$

${ }^{57}$ Ibid., p. 206, v. 87-89.

${ }^{58}$ Ibid., p. 208, v. 177-185. 
Dans ces derniers vers nous retrouvons, sous forme de moralité explicite, le thème que nous avons rencontré dans Charlot le Juif et aussi, si l'on veut, dans le Couronnement de Renart : «tel est pris qui croyait prendre ». «Qui barat quier, baraz li vient ${ }^{60}$ avait dit Rutebeuf ; ici, de façon plus imagée, Colin Malet dit : «Qui merde brace, merde boive $»^{61}$. Encore une fois, nous trouvons la merde du côté du paysan et, de plus, associée à une mésalliance. Il faut peut-être rapprocher Jouglet d'un autre fabliau, Berengier au lonc $\mathrm{cul}^{62}$, où un riche paysan, marié à une noble pauvre, se trouve, à la fin du texte, contraint de se plier à une humiliation scatologique : embrasser le derrière de sa femme déguisée en chevalier. Ici, les excréments rabaissent en même temps le jongleur, qui voulait conchier et qui est conchié, et le paysan, dont la bêtise est sans borne. Nous pouvons enfin remarquer que c'est la deuxième fois qu'on voit apparaître un jongleur dans notre petit corpus scatologique.

Pour conclure, dans nos récits les excréments nous ont paru associés à certains thèmes que nous nous contentons de citer, sans les développer davantage. Tout d'abord, ils sont liés à la ruse et à l'humiliation, et aux multiples combinaisons de ces deux notions. Dans trois cas nous avons vu que cette ruse repose sur l'utilisation de la matière fécale comme médecine, et nous avons argué qu'il s'agit peut-être de réminiscences de pratiques de la médecine populaire. La matière fécale est liée de manière très évidente à une classe sociale, celle des paysans, à travers un rapport métonymique (le paysan est conchié) aussi bien que métaphorique (le paysan est une image du fumier). Cette interprétation est étayée par ces récits à contenu anal ou scatologique qui mettent en scène une mésalliance, tels que Berangier au

\footnotetext{
${ }^{59}$ Ibid., p. 214, v. 416-420.

${ }^{60}$ Rutebeuf, Le Pet au Vilain, éd. cit., p. 259, v. 132.

${ }^{61}$ Jouglet, in N.R.C.F., éd. cit., p. 209, v. 208.

${ }^{62}$ Berengier au lonc cul, in N.R.C.F., éd. cit., t. IV, p. 245-277.
} 
lonc cul et Jouglet. Outre des paysans, nous avons vu à deux reprises des jongleurs associés à cette matière et il est possible que cette présence soit le témoignage du discrédit dans lequel on tenait cette catégorie professionnelle ${ }^{63}$. Enfin, dans deux textes, un seigneur est conchié. Pourtant, la hiérarchie médiévale n'est véritablement inversée que dans l'un des deux, Trubert. Dans Charlot le Juif la matière fécale rabaisse le petit seigneur en même temps que le jongleur et, en outre, la ruse scatologique sanctionne l'absence du devoir social nobiliaire qu'est la largesse. Seul un texte de notre corpus, donc, nous permet de repérer ce «réalisme grotesque » qui, selon la théorie bakhtinienne, est un principe littéraire et social dont la fonction est le rabaissement de tout ce qui est élevé et noble.

Malgré la place qu'un ouvrage comme celui de Bakhtine fait à la scatologie dans la littérature médiévale française, force est de constater que les savants n'ont pas suivi les riches pistes que l'érudit russe avait généreusement ouvertes dans son livre. D'ailleurs, la réception par la critique littéraire de l'ouvrage sur la culture populaire de Rabelais a peutêtre fini par devenir un horizon borné pour les études dans ce domaine, plutôt qu'une incitation à des recherches originales. En effet, la critique américaine et anglo-saxonne, de façon plus ou moins explicite, s'appuie à l'heure actuelle de façon massive sur la vision «subversive » bakhtinienne $\mathrm{du}$ «bas corporel ». Cela conduit parfois à des interprétations quelque peu excessives, comme celle qui verrait dans le fabliau La Crote une parodie de

\footnotetext{
${ }^{63}$ Dans un sermon, nous trouvons cette affirmation qui se réfère aux jongleurs, citée par Léon Gautier, Les Épopées françaises, Paris, Welter, 1878-1892, 4 vol., vol. 2, p. 197 : « libentius ponunt linguam ad stercora mundi quam ad lapides pretiosos coeli » («ils posent plus volontiers leur langue sur les excréments du monde que sur les précieux pavés du ciel »). Depuis plusieurs années, on essaie cependant de nuancer l'affirmation selon laquelle les jongleurs au Moyen Âge ont été dévalorisés et marginalisés. Pour une étude récente, voir Silvère MENEGALDO, Le Jongleur dans la littérature narrative des XII et XIII ${ }^{e}$ siècles: du personnage au masque, Paris, Champion, 2005.
} 
la transsubstantiation ${ }^{64}$. Or, parmi les textes scatologiques que nous avons vus dans cette rapide étude, il n'y en a qu'un, Trubert, qui met en scène une explicite subversion de l'échelle hiérarchique ou des idées dominantes.

En revanche, une véritable et explicite révolution dans la vision de l'homme sur les excréments apparaît chez un auteur de la Renaissance qui a été peut-être le premier à donner une vraie noblesse à la matière la plus vile. Ce n'était pas rien, pour Montaigne, de comparer ses Essais à de la merde : «Ce sont icy, des excremens d'un vieil esprit, dur tantost, tantost lache, et toujours indigeste ${ }^{65}$. C'est que lui, l'écrivain-philosophe, avait compris la charge démocratique et subversive des excréments : «Et les Roys et les philosophes fientent, et les dames aussi ${ }^{66}$.

${ }^{64}$ Cf. Sheila J. NAYAR, «Coprus [sic] Christi... », art. cit.

${ }^{65}$ Michel de Montaigne, Essais, Paris, Flammarion, 1979, t. III, chap. IX, « De la vanité », p. 159.

${ }^{66}$ Ibid., chap. XIII, « De l'expérience », p. 296. 